\title{
The Efficacy of Brimonidine in Preventing Intraocular Pressure Elevation in the Provocative Test for Primary Angle-Closure Glaucoma
}

\author{
YUH-FANG CHEN,${ }^{1,2}$ POR T. HUNG,${ }^{1}$ JUI-WEI HSIEH, ${ }^{1}$ \\ JEAN SHEIN, ${ }^{3}$ and CHUHSING K. HSIAO ${ }^{4}$ \\ ${ }^{1}$ Department of Ophthalmology, National Taiwan University Hospital, Taipei, Taiwan \\ ${ }^{2}$ Department of Health, Taipei Hospital Capital Branch, Taipei, Taiwan \\ ${ }^{3}$ New York University School of Medicine, New York, New York \\ ${ }^{4}$ Division of Biostatistics, Institute of Epidemiology, National Taiwan University, Taipai, Taiwan
}

\begin{abstract}
The purpose of this study was to evaluate the efficacy of $0.2 \%$ brimonidine tartrate in preventing intraocular pressure (IOP) elevation in the dark-prone provocative test for primary angle-closure glaucoma (PACG). Twenty-two eyes from 22 patients with angleclosure glaucoma were enrolled in this study. Each of the selected eyes had previously tested positive in a recent dark-prone test. One drop of $0.2 \%$ brimonidine tartrate was then instilled in each eye 2 hours prior to a second dark-prone test. An IOP elevation of greater than $8 \mathrm{mmHg}$ was regarded as a positive result. The IOP elevation in the first dark-prone test was $11.91 \pm 5.17$ (range: $5.7-27.3$ ) $\mathrm{mmHg}$, while the IOP only increased $5.70 \pm$ 2.96 (range: $2.9-12.2$ ) $\mathrm{mmHg}$ in the second dark-prone test that was pre-treated with $0.2 \%$ brimonidine tartrate $(\mathrm{p}<0.001)$. A significant difference was also noted in the pretest IOP $(15.59 \pm 3.86 \mathrm{mmHg}$ vs. $13.33 \pm 3.65 \mathrm{mmHg}, \mathrm{p}=0.008)$ as well as in the posttest IOP $(27.62 \pm 7.27 \mathrm{mmHg}$ vs. $19.03 \pm 3.50 \mathrm{mmHg}, \mathrm{p}<0.001)$ in the two sequential dark-prone tests. All but three of the initially positive dark-prone tests $(86.46 \%)$ converted to negative tests after pre-treatment with brimonidine. There was a significant effect of $0.2 \%$ brimonidine tartrate in the prevention of IOP elevation in PACG patients previously found to test positive in the dark-prone provocative test.
\end{abstract}

\section{INTRODUCTION}

The incidence of primary angle-closure glaucoma (PACG) is higher in Asian populations, especially among the ethnic Chinese (1). Among these populations, PACG is more frequently chronic and 
has few symptoms. It is also characterized by a gradual progression to angle-closure even after the pupillary-block mechanism has been eliminated by successful iridectomy (2). Therefore, the mechanisms of intraocular pressure (IOP) elevation in PACG are complicated, and it is important to investigate the pathogenesis of the mechanical forces leading to angle-closure. Since the pathophysiology of PACG is so different from primary open-angle glaucoma (POAG), the IOP measurements, optic nerve evaluations, and visual function tests generally accepted for POAG detection are not suitable for early detection of PACG.

On the other hand, the provocative test is an important technique that may allow clinicians to predict if an asymptomatic PACG patient with a normal IOP will develop angle-closure and IOP elevation. Since the pathophysiologies of POAG and PACG are different, the treatment modalities of these two entities of glaucoma also differ. For PACG, medications that can effectively prevent angle-closure and IOP elevation are more important than those for daily IOP control, which are required in POAG. Hung et al advocated that pilocarpine has a significant effect in preventing the rise of IOP after dark-prone tests in PACG (3). In this study, $0.2 \%$ brimonidine tartrate was used prior to a darkprone test to evaluate its efficacy in preventing IOP elevation after provocation.

\section{MATERIALS AND METHODS}

We enrolled 22 angle-closure glaucoma patients who previously had at least one positive darkprone test. They were classified into groups based on the morphology of their glaucoma. Diagnostic criteria for the acute glaucoma category included elevated IOP above $25 \mathrm{mmHg}$ associated with angle closure. For chronic PACG, there should be an occludable angle with or without peripheral anterior synechiae, previous positive provocative tests, biometric evidence, and glaucomatous optic neuropathy. All eyes with chronic PACG were quiet and the IOP was below $20 \mathrm{mmHg}$ prior to the dark-prone test. Patients who had persistently high IOPs in spite of patent iridectomies were classified as having post-iridectomy glaucoma (PIG). Plateau iris configuration was defined as the presence of an occludable angle in combination with a normal-depth anterior chamber (4).

The first modified dark-prone test was performed without pre-medication after washing out for previous medications. The patients remained in a sitting position with their faces down on the desk for 1.5 hours in a dark room. Darkness was enhanced with opaque goggles, and patients were instructed to keep both eyes open. An IOP elevation of more than $8 \mathrm{mmHg}$ or a post-test IOP of more than $25 \mathrm{mmHg}$ was considered positive. Those who had at least one positive test in one eye were enrolled in the study, and a subsequent dark-prone test was performed. For the subsequent test, one drop of $0.2 \%$ brimonidine was instilled in both eyes two hours prior to the test. A modified dark-prone test of the same duration was then performed again.

The eye with the positive result in the first dark-prone test was selected for study analysis. If both eyes were positive, the one with more elevated IOP was chosen. If both eyes had equal IOP elevation, the one with higher post-test IOP was analyzed. If post-test IOP was equal between two eyes, the right eye was defined as the study eye. The results were expressed as mean \pm standard deviation. A paired student's t-test was used for the result analysis, and $\mathrm{p}<0.001$ was defined as being statistically significant.

\section{RESULTS}

Twenty-two eyes from 22 patients were analyzed. There were 3 eyes with acute PACG, 11 with chronic PACG, 5 with PIG, and 3 with plateau iris configuration. The patients consisted of 6 men and 16 women, and their average age was $58.55 \pm 7.99$ (range from 42 to 76) years old.

The results of the dark-prone tests are summarized in Table 1. The IOP elevation in the first, non-medicated dark-prone test was $11.91 \pm 5.17$ (range from 5.7 to 27.3 ) $\mathrm{mmHg}$, while the IOP only 


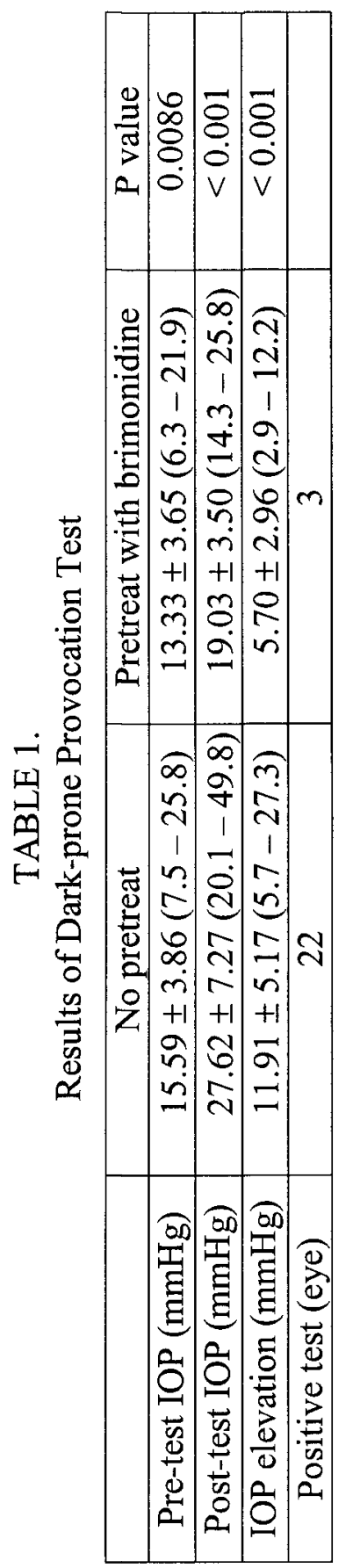


increased $5.70 \pm 2.96$ (range from 2.9 to 12.2 ) $\mathrm{mmHg}$ in the dark-prone test with the $0.2 \%$ brimonidine pre-treatment $(\mathrm{p}<0.001)$. A significant difference was also noted between the post-test IOP $(27.62 \pm 7.27 \mathrm{mmHg}$ vs. $19.03 \pm 3.50 \mathrm{mmHg}, \mathrm{p}<0.001)$ in the two sequential dark-prone tests. The pre-test IOP was also lower when pre-treated with brimonidine; however, it did not reach the level of statistical significance $(\mathrm{p}=0.0086)$. All but three of the initially positive dark-prone tests $(86.46 \%)$ converted to negative tests after pre-treatment with brimonidine. The $95 \%$ confidence interval ranged from 0.72 to 1.00 .

\section{DISCUSSION}

Brimonidine is a newly approved, highly selective alpha-2 adrenergic agonist. It decreases IOP by both suppressing aqueous humor inflow and increasing uveoscleral outflow (5). In a three-month study, mean peak IOPs with twice-daily dosing of $0.2 \%$ brimonidine were reduced by $24 \%$, and the IOP reduction at trough was $15 \%$ in patients with ocular hypertension (6). Furthermore, $0.5 \%$ brimonidine has been proven to successfully prevent laser-induced acute IOP spikes and to reduce the mean IOP after argon trabeculoplasty (7). In this study, we reported a significant effect of $0.2 \%$ brimonidine tartrate in the prevention of IOP elevation in dark-prone provocation in patients with angle-closure glaucoma. Both the IOP elevation and post-dark-prone IOP were significantly decreased in the group pre-treated with $0.2 \%$ brimonidine tartrate $(\mathrm{p}<0.001)$. Furthermore, $86.46 \%$ (19 in 22) of the PACG patients that initially tested positive in the dark-prone test later tested negative after using brimonidine.

Mechanisms of PACG can traditionally be classified as pupillary block and non-pupillary block types. However, it has been well recognized that in most instances these two types were mixed $(8,9)$. Hung et al reported that $59.5 \%$ of 37 iridectomized eyes of PACG patients tested positive in the darkprone provocative test. These eyes were previously assumed to be free from pupillary block (3). Such iridectomized eyes were labeled residual glaucoma or post-iridectomy glaucoma (PIG) (10). Mechanisms involved in non-pupillary block PACG or PIG include the plateau iris-like angle crowding mechanism, malignant glaucoma-like ciliary block mechanism, peripheral anterior synechiae, trabecular dysfunction, and/or lens factors (3). In the Chinese, a slow progression of angle-closure or the creeping angle is another important mechanism responsible for PIG (2).

Since mechanisms of PACG are different from those of POAG, parameters commonly used for the early detection of POAG, such as IOP, optic nerve assessment, and visual field changes, are not proper for the early detection of PACG. For this purpose, various provocative tests have been introduced. These include the dark-room, prone-position, dark-prone, and pharmacological pupillary dilation tests (11). The positive rates and predictive values of these tests are widely varied. However, generally speaking, negative results cannot exclude the possibility of PACG; only positive results are significant.

Aside from detecting PACG in its early stages, provocative tests also have an important role in evaluating the efficacy of PACG medications. Hung et al demonstrated that topical application of $1 \%$ pilocarpine prior to a dark-prone test significantly lowered rates of positive tests $(15 \%)$ when compared to those pre-treated with $0.25 \%$ timolol $(68 \%)$ or those without pre-treatment $(100 \%)$ in 31 iridectomized PACG eyes. They also showed that after $1 \%$ pilocarpine pre-treatment in 8 previously positive dark-prone tests, only 3 (38\%) remained positive (2). In our study, $86.46 \%$ of the positive dark-prone provocative tests converted into negative results after $0.2 \%$ brimonidine pre-treatment in 22 PACG eyes.

\section{ACKNOWLEDGMENTS}

This study was supported, in part, by the National Science Council, Executive Yuan, ROC, grants number NSC89-2314-B-002-580 and NSC89-2314-B-002-581. 


\section{REFERENCES}

1. Hung, P.T. Angle-closure glaucoma. J. Clin. Ophthalmol. 8:27-31, 1990.

2. Hung, P.T. and Chen, Y.C. Medical therapy in primary angle-closure glaucoma: a model for evaluation of its efficacy. In Glaucoma Update, Krieglstein, G.K., ed., New York: Springer, 2000, pp. 169-172.

3. Hung, P.T. Provocation and medical treatment in post-iridectomy glaucoma. J. Ocul. Pharmacol. 6:279-283, 1990.

4. Hoskins, H.D. Jr. and Kass, M.A. Primary angle closure glaucoma without pupillary block (plateau iris). In Diagnosis and Therapy of the Glaucoma, $6^{\text {th }}$ ed., Hoskins, H.D. Jr. and Kass, M.A., eds., St Louis: CV Mosby Co., 1989, pp. 241-242.

5. Toris, C.B., Gleason, M.L., Camras, C.B., and Yablonski, M.E. Effects of brimonidine on aqueous humor dynamics in human eyes. Arch. Ophthalmol. 113:1514-1517, 1995.

6. Serle, J.B. A comparison of the safety and efficacy of twice daily brimonidine $0.2 \%$ versus betaxolol $0.25 \%$ in subjects with elevated intraocular pressure. The brimonidine Study Group III. Surv. Ophthalmol. 41(Suppl 1):S39-S47, 1996.

7. Anonymous (The Brimonidine-ALT Study Group). Effect of brimonidine $0.5 \%$ on intraocular pressure spikes following $360^{\circ}$ argon laser trabeculoplasty. Ophthalmic Surg. Lasers 26: 404-409, 1995.

8. Hung, P.T. and Chou, L.H. Provocation and mechanism of angle-closure glaucoma after iridectomy. Arch. Ophthalmol. 97:1862-1864, 1979.

9. Hoskins, H.D. Jr. and Kass, M.A. Introduction and classification of the glaucoma. In Diagnosis and Therapy of the Glaucoma, $6^{\text {th }}$ ed., Hoskins, H.D. Jr. and Kass, M.A., eds., St Louis: CV Mosby Co., 1989, pp. 2-9.

10. Forbes, M. and Becker, B. Iridectomy in advanced angle closure glaucoma. Am. J. Ophthalmol. 57:57-62, 1964.

11. Hyams, S.W., Friedman, Z., and Meumann, E. Elevated pressure in the prone position. A new provocative test for angle-closure glaucoma. Am. J. Ophthalmol. 66:661-672, 1968.

Received: May 16, 2001

Accepted for Publication: July 26, 2001

Reprint Requests: Por T. Hung, Ph.D.

Department of Ophthalmology

National Taiwan University Hospital

No. 7, Chung-Shan South Rd.

Taipei, Taiwan ROC

E-mail: portying@ha.mc.ntu.edu.tw 